Mathematical Research Letters 5, 1-12 (1998)

\title{
NONABELIAN INTEGRABLE SYSTEMS, QUASIDETERMINANTS, AND MARCHENKO LEMMA
}

\author{
Pavel Etingof, Israel Gelfand, and Vladimir Retakh
}

\begin{abstract}
We find explicit (multisoliton) solutions for nonabelian integrable systems such as periodic Toda field equations, Langmuir equations, and Schrödinger equations for functions with values in any associative algebra. The solution for nonabelian Toda field equations for root systems of types $A, B, C$ was expressed by the authors in [EGR] using quasideterminants introduced and studied in [GR1GR4]. To find multisoliton solutions of periodic Toda equations and other nonabelian systems we use a combination of these ideas with important lemmas which are due to Marchenko [M].
\end{abstract}

\section{Introduction}

Let $R$ be an algebra. A map $\partial: R \rightarrow R$ is called a derivation of $R$ if $\partial(a b)=(\partial a) b+a \partial b$. Suppose that $R$ is an algebra with unit, and $\partial_{1}, \partial_{2}$ two derivations of $R$ commuting with each other.

Definition 1. One says that invertible elements $g_{i}, i=0,1, \ldots, n-1$ satisfy the n-periodic Toda equations if

$$
\partial_{1}\left(\left(\partial_{2} g_{k}\right) g_{k}^{-1}\right)=g_{k} g_{k-1}^{-1}-g_{k+1} g_{k}^{-1}, k \in \mathbb{Z} / n \mathbb{Z}
$$

In this paper we construct solutions of nonabelian periodic Toda systems and other integrable systems using a combination of the theory of quasideterminants developed in [GR1, GR2, GR3, GR4] and Marchenko approach [M] to integrable systems of differential equations on functions with values in operator algebras.

Let $Q$ be a not necessarily commutative algebra with derivations $\partial_{1}, \ldots, \partial_{m}$ and automorphisms $\alpha_{1}, \ldots, \alpha_{s}$. Marchenko approach says that when an element $\Gamma \in Q$ satisfies a certain system of linear differential equations connecting the $\partial$ 's, $\alpha$ 's and $\Gamma$, then its (noncommutative) logarithmic derivative $\left(\partial_{1} \Gamma\right) \Gamma^{-1}$ satisfies a system of nonlinear equations.

Unfortunately Marchenko's book is unavailable in English; we will reproduce parts of its material for the reader's convenience.

Note that nonabelian Toda equations for the root system $A_{n-1}$ were introduced by Polyakov (see $[\mathrm{Kr}]$ ). Nonabelian Toda lattice for functions of one variable appeared in $[\mathrm{PC}],[\mathrm{P}]$. Krichever $[\mathrm{Kr}]$ constructed algebraic-geometric

Received July 15, 1997. 
solutions for the periodic nonabelian Toda lattices for $N \times N$-matrix-valued functions.

Remark. Our construction of multisoliton solutions is similar to the ideas of higher rank solitons proposed by Krichever and Novikov at the end of seventies (see e.g. $[\mathrm{KN}]$ ).

\section{Quasideterminants and Wronski Matrices}

Quasideterminants were introduced in [GR1], as follows. Let $X$ be an $m \times m$ matrix over a ring $A$. For any $1 \leq i, j \leq m$, let $r_{i}(X), c_{j}(X)$ be the i-th row and the j-th column of $X$. Let $X^{i j}$ be the submatrix of $X$ obtained by removing the i-th row and the j-th column from $X$. For a row vector $r$ let $r^{(j)}$ be $r$ without the j-th entry. For a column vector $c$ let $c^{(i)}$ be $c$ without the i-th entry. Assume that $X^{i j}$ is invertible. Then the quasideterminant $|X|_{i j} \in A$ is defined by the formula

$$
|X|_{i j}=x_{i j}-r_{i}(X)^{(j)}\left(X^{i j}\right)^{-1} c_{j}(X)^{(i)},
$$

where $x_{i j}$ is the $i j$-th entry of $X$.

Let $Q$ be an algebra with unit, and $\partial: Q \rightarrow Q$ its derivation. For $f \in Q$ denote $\partial^{k} f$ by $f^{(k)}, k=1,2, \ldots$ and set $f^{(0)}=f$. For elements $f_{1}, \ldots, f_{N}$ denote by $W=W\left(f_{1}, \ldots, f_{N}\right)$ their Wronski matrix,

$$
W=\left(\begin{array}{ccc}
f_{1} & \ldots & f_{N} \\
\partial f_{1} & \ldots & \partial f_{N} \\
. & \ldots & . \\
\partial^{N-1} f_{1} & \ldots & \partial^{N-1} f_{N}
\end{array}\right)
$$

Set

$$
\begin{aligned}
\partial W & =\left(\partial f_{j}^{(k-1)}\right)=\left(f_{j}^{(k)}\right), \\
W^{i} & =\left(f_{j}^{(m)}\right), m=0, \ldots, \hat{i}, \ldots, N, j=1, \ldots, N .
\end{aligned}
$$

Suppose that $W$ is invertible and consider the matrix $X=(\partial W) W^{-1}=\left(\eta_{p q}\right)$. Let $\delta_{p q}$ be the Kronecker symbol.

Proposition 1. For $p=1, \ldots, N-1$

i)

$$
\eta_{p q}=\delta_{p+1, q},
$$

and for $q=1,2, \ldots, N$

ii)

$$
\eta_{N q}=\left|W^{q}\right|_{N N}|W|_{q N}^{-1} .
$$

Proof. By our construction $\partial W=X W$. It is easy to see that this identity is satisfied if and only if i) is valid for $p=1, \ldots, N-1$, and for $q=1, \ldots, N$

$$
f_{q}^{(N)}=\eta_{N 1} f_{q}^{(0)}+\cdots+\eta_{N N} f_{q}^{(N-1)} .
$$


Formulas ii) follow from Cramer rules [GR1, GR2, GR3, GR4] applied to the right system of linear equations (2).

Remark. Consider the $(N+1) \times N$-matrix

$$
\tilde{W}=\left(\begin{array}{ccc}
f_{1} & \ldots & f_{N} \\
\partial f_{1} & \ldots & \partial f_{N} \\
\ddot{n} & \ldots & \ddot{~} \\
\partial^{N} f_{1} & \ldots & \partial^{N} f_{N}
\end{array}\right) .
$$

Then $\eta_{q N}$ is the right quasi-Plücker coordinate $r_{N q}^{1 \ldots \hat{i} \ldots N-1}(\tilde{W})$ defined in [GR4].

Square matrices $\left(\eta_{p q}\right)$ satisfying Property i) in Proposition 1 are often called Frobenius cells.

\section{Marchenko Lemma}

Let $R$ be a ring with unit, $\partial_{i}: R \rightarrow R, i=1,2$ its derivations, and $\alpha: R \rightarrow R$ an isomorphism, such that $\partial_{i} \alpha=\alpha \partial_{i}$ for $i=1,2$. Let also $\Gamma, A \in R$ be invertible elements such that $\partial_{i} A=0, i=1,2, \partial_{1}\left(\partial_{2} \Gamma\right)=\Gamma$, and $\partial_{2} \Gamma=\alpha(\Gamma) A$. Set $\gamma=\left(\partial_{2} \Gamma\right) \Gamma^{-1}$. The following lemma was proved in $[\mathrm{M}]$.

\section{Marchenko Lemma.}

$$
\partial_{1}\left(\partial_{2} \gamma\right) \gamma^{-1}=\gamma \alpha^{-1}\left(\gamma^{-1}\right)-\alpha(\gamma) \gamma^{-1}
$$

Proof. We will use the identity $\delta\left(a^{-1}\right)=-a^{-1}(\delta a) a^{-1}$ for an invertible element $a \in R$ and a derivation $\delta: R \rightarrow R$. One could write $\gamma$ as $\gamma=\alpha(\Gamma) A \Gamma^{-1}$. Then

$$
\begin{aligned}
\partial_{2} \gamma & =\left(\partial_{2} \alpha(\Gamma)\right) A \Gamma^{-1}-\alpha(\Gamma) A \Gamma^{-1}\left(\partial_{2} \Gamma\right) \Gamma^{-1} \\
& =\alpha^{2}(\Gamma) \alpha(A) A \Gamma^{-1}-\alpha(\Gamma) A \Gamma^{-1} \alpha(\Gamma) A \Gamma^{-1} .
\end{aligned}
$$

Then

$$
\left(\partial_{2} \gamma\right) \gamma^{-1}=\alpha^{2}(\Gamma) \alpha(A) \alpha(\Gamma)^{-1}-\alpha(\Gamma) A \Gamma^{-1} .
$$

Note that $\left(\partial_{1} \alpha(\Gamma)\right) A=\partial_{1}(\alpha(\Gamma) A)=\partial_{1}\left(\partial_{2} \Gamma\right)=\Gamma$. So, $\partial_{1}(\alpha(\Gamma))=\Gamma A^{-1}$, and $\partial_{1}(\Gamma)=\alpha^{-1}(\Gamma) \alpha^{-1}(A)^{-1}$. From (4) it follows that

$$
\begin{aligned}
\partial_{1}\left(\partial_{2}(\gamma) \gamma^{-1}\right)= & \alpha(\Gamma) \alpha(A)^{-1} \alpha(A) \alpha(\Gamma)^{-1}-\alpha^{2}(\Gamma) \alpha(A) \alpha(\Gamma)^{-1} \Gamma A^{-1} \alpha(\Gamma)^{-1} \\
& -\Gamma A^{-1} A \Gamma^{-1}+\alpha(\Gamma) A \Gamma^{-1} \alpha^{-1}(\Gamma) \alpha^{-1}(A)^{-1} \Gamma^{-1} \\
= & -\alpha^{2}(\Gamma) \alpha(A) \alpha(\Gamma)^{-1} \Gamma A^{-1} \alpha(\Gamma)^{-1} \\
& +\alpha(\Gamma) A \Gamma^{-1} \alpha^{-1}(\Gamma) \alpha^{-1}(A)^{-1} \Gamma^{-1}
\end{aligned}
$$

On the other hand,

$$
\begin{aligned}
\gamma \alpha^{-1}\left(\gamma^{-1}\right)-\alpha(\gamma) \gamma^{-1}= & \alpha(\Gamma) A \Gamma^{-1} \alpha^{-1}(\Gamma) \alpha^{-1}(A)^{-1} \Gamma^{-1} \\
& -\alpha^{2}(\Gamma) \alpha(A) \alpha(\Gamma)^{-1} \Gamma A^{-1} \alpha(\Gamma)^{-1}
\end{aligned}
$$




\section{Specialization of Marchenko Lemma}

We will apply Marchenko Lemma to the following data. Let $Q$ be an algebra, and let $\partial_{i}: Q \rightarrow Q, i=1,2$ be derivations of $Q$ commuting with each other. Set $R=Q^{n}=\left\{\left(w_{0}, \ldots, w_{n-1}\right)\right\}$ be an algebra with coordinate-wise multiplication and coordinate-wise derivations induced by $\partial_{i}, i=1,2$. These derivations will be denoted by the same letters. To construct an isomorphism $\alpha: R \rightarrow R$, let us suppose that indices $0,1, \ldots$, $n-1 \in \mathbb{Z} / n \mathbb{Z}$ and set $\alpha\left(\left(w_{k}\right)\right)=\left(w_{k+1}\right)$. Then $\alpha^{-1}\left(\left(w_{k}\right)\right)=\left(w_{k-1}\right)$. Let $A_{k} \in Q, k=0,1, \ldots, n-1$ be elements such that for $k=0,1, \ldots, n-1$

$$
\partial_{i} A_{k}=0, i=1,2
$$

and $\left(w_{k}\right)$ be such that

$$
\partial_{2} w_{k}=w_{k+1} A_{k}, \partial_{1} w_{k}=w_{k-1} A_{k-1}^{-1} .
$$

Set

$$
\gamma_{k}=\left(\partial_{2} w_{k}\right) w_{k}^{-1}, k=0,1, \ldots, n-1 .
$$

Then Marchenko Lemma implies

\section{Proposition 2.}

$$
\partial_{1}\left(\left(\partial_{2} \gamma_{k}\right) \gamma_{k}^{-1}\right)=\gamma_{k} \gamma_{k-1}^{-1}-\gamma_{k+1} \gamma_{k}^{-1}, k=0,1, \ldots, n-1 .
$$

\section{Frobenius cells}

We will apply the results of subsection 3 to Frobenius cells. Consider matrices

$$
K=\left(\mu_{p q}\right), \quad L=\left(\nu_{p q}\right), \quad \text { and } \quad Y=\left(y_{p q}\right), \quad p, q=1, \ldots, N,
$$

over an algebra with unit. Suppose that matrices $K$ and $L$ are Frobenius cells, i.e. that $\mu_{p q}=\nu_{p q}=\delta_{p+1 q}$ for $p=1, \ldots, N-1, q=1, \ldots, N(\delta$ is the Kronecker symbol).

Lemma 3. Suppose that $K=Y L$ and that $\nu_{N 1}$ is invertible. Then $y_{p q}=\delta_{p q}$ for $p=1, \ldots, N-1, q=1, \ldots, N$ and

$$
\begin{aligned}
y_{N q} & =\mu_{N, q+1}-\mu_{N 1} \nu_{N 1}^{-1} \nu_{N, q+1}, q=1, \ldots, N-1, \\
y_{N N} & =\mu_{N 1} \nu_{N 1}^{-1} .
\end{aligned}
$$

Proof. Straightforward.

Suppose that under conditions of Lemma $3 \gamma_{k}=\left(g_{k, p q}\right), p, q=1, \ldots, N$, is a Frobenius cell for $k=0,1, \ldots, n-1$. Then Lemma 3 immediately implies

Corollary 4. Elements $g_{k}=g_{k, N 1}, k=0,1, \ldots, n-1$ satisfy the Toda periodic system (1). 


\section{Solutions of the Toda system}

Let $Q$ be an algebra with unit, and let $\partial_{1}, \partial_{2}: Q \rightarrow Q$ be two commuting derivations. Let $f_{i j}, a_{i j} i \in \mathbb{Z} / n \mathbb{Z}, j=1, \ldots, N$ be elements in $Q$ such that for all $i, j a_{i j}$ are invertible and

$$
\begin{gathered}
\partial_{1} a_{i j}=\partial_{2} a_{i j}=0, \\
\partial_{1} f_{i j}=f_{i-1 j} a_{i j}^{-1}, \\
\partial_{2} f_{i j}=f_{i+1 j} a_{i+1 j} .
\end{gathered}
$$

Consider Wronski matrices $W_{k}=W\left(f_{k 1}, \ldots, f_{k N}\right)$, using $\partial_{2}$ as a derivation and diagonal matrices

$$
A_{k}=\operatorname{diag}\left(a_{k 1}, \ldots, a_{k N}\right), \quad \text { for } \quad k=0,1,2, \ldots, n-1 .
$$

Suppose that all these matrices are invertible and the quasideterminants $\left|\partial_{2} W_{k}\right|_{N N}$ are defined. Set

$$
g_{k}=\left|\partial_{2} W_{k}\right|_{N N}\left|W_{k}\right|_{1 N}^{-1}, \quad k=0,1, \ldots, n-1 .
$$

Theorem 5. Elements $g_{k}, k=0,1, \ldots, n-1$ satisfy Toda system (1).

Proof. Set

$$
\gamma_{k}=\left(\partial_{2} W_{k}\right) W_{k}^{-1}, \quad k=0,1, \ldots, n-1 .
$$

Then apply Proposition 2, Lemma 3, and Proposition 1.

\section{Toda systems over formal series of two variables}

To construct sets of elements $f_{i j}, a_{i j}$ satisfying the conditions of subsection 5 consider the algebra $Q=S[[u, v]]$ of formal series of commutative variables $u, v$ over a not necessarily commutative algebra $S$.

Set $\partial_{1}=\frac{\partial}{\partial u}, \partial_{2}=\frac{\partial}{\partial v}$. Choose any elements $a_{i j} \in S$. Let us construct $f_{i j}$. Consider the diagonal matrices

$$
a_{j}=\operatorname{diag}\left(a_{0 j}, \ldots, a_{n-1 j}\right), \quad j=1, \ldots, N .
$$

Let $R$ be the matrix of permutation $\sigma(1, \ldots, n)=(2, \ldots, n, 1)$. Set $R_{j}=R a_{j}$, $j=1, \ldots, N$. Consider exponentials $e_{j}=e^{R_{j}^{-1} u+R_{j} v}$ as formal $n \times n$-matrix series of $u$ and $v, j=1, \ldots, N$. For $j=1, \ldots, N$ take any $1 \times n$-matrix $p_{j}$ (a row vector) over $S$ and set $f_{j}=p_{j} e_{j}, j=1, \ldots, N$. Denote elements of $f_{j}$ by $f_{0 j}, \ldots, f_{n-1 j}$.

Proposition 6. Suppose that derivatives $\partial_{1}, \partial_{2}$ are defined as above. Then elements $f_{i j}, i=0,1 \ldots, n-1, j=1, \ldots, N$ satisfy equalities (6) and (7).

Proof. It is enough to note that $\frac{\partial}{\partial u} e_{j}=e_{j} R_{j}^{-1}$, and $\frac{\partial}{\partial v} e_{j}=e_{j} R_{j}$ for $j=$ $1, \ldots, N$.

Remark. Thus, we have constructed a family of solutions of the periodic Toda equations which are parametrized by $2 n N S$-valued parameters ( $n N$ elements of the $n$-dimensional vectors $p_{1}, \ldots, p_{N}$, and $n N$ elements $a_{i j}$ ). 


\section{Example: the sine-Gordon equation}

In the commutative case the sine-Gordon equation is obtained from the 2periodic Toda system. We consider here the 2-periodic nonabelian Toda system. In the notations of subsections 5,6 set

$$
f_{i j}=p_{j} e^{a_{j}^{-1} u+a_{j} v}+(-1)^{i} q_{j} e^{-a_{j}^{-1} u-a_{j} v}, i \in \mathbb{Z} / 2 \mathbb{Z}, j=1, \ldots, N
$$

with $p_{j}, q_{j}, a_{j} \in S$. Then $\left(f_{i j}\right)$ satisfy equations (6), (7) with $a_{i j}=a_{j}$.

Set $N=1$ and omit the index $j=1$ in our notations. According to Theorem 5

$$
\gamma_{i}=\left(p-(-1)^{i} q e^{-2 a^{-1} u-2 a v}\right) a\left(p+(-1)^{i} q e^{-2 a^{-1} u-2 a v}\right)^{-1}, i \in \mathbb{Z} / 2 \mathbb{Z}
$$

are solutions of the 2-periodic Toda system.

Remark. If $S$ has an involutive antiautomorphism $*$ then $\gamma_{0}=\gamma_{1}^{*}$ for appropriate $a, p, q$. Then $\gamma=\gamma_{0}$ satisfies the noncommutative sine-Gordon equation

$$
\partial / \partial u\left((\partial \gamma / \partial v) \gamma^{-1}\right)=\gamma\left(\gamma^{*}\right)^{-1}-\gamma^{*} \gamma^{-1}
$$

If $S=\mathbb{C}$ and $*$ is the complex conjugation then substitution $\gamma=e^{i f}$ leads us to the sine-Gordon equation.

For $N=2$ formulas (8) imply

$$
\begin{aligned}
\gamma_{i}= & \left(f_{i 2} a_{2}^{2}-f_{i 1} a_{1} f_{i-1,1}^{-1} f_{i-1,2} a_{2}\right)\left(f_{i 2}-f_{i 1} a_{1}^{-1} f_{i-1,1}^{-1} f_{02} a_{2}\right)^{-1} \\
= & \left\{\left(p_{2}+(-1)^{i} q_{2} \eta_{2}\right) a_{2}\right. \\
& \left.\quad-\left(p_{1}+(-1)^{i} q_{1} \eta_{1}\right) a_{1}\left(p_{1}-(-1)^{i} q_{1} \eta_{1}\right)^{-1}\left(p_{2}-(-1)^{i} q_{2} \eta_{2}\right)\right\} \\
& \times\left\{\left(p_{2}+(-1)^{i} q_{2} \eta_{2}\right) a_{2}^{-1}\right. \\
& \left.\quad-\left(p_{1}+(-1)^{i} q_{1} \eta_{1}\right) a_{1}^{-1}\left(p_{1}-(-1)^{i} q_{1} \eta_{1}\right)^{-1}\left(p_{2}-(-1)^{i} q_{2} \eta_{2}\right)\right\}^{-1},
\end{aligned}
$$

where $\eta_{j}=e^{-2 a_{j}^{-1} u-2 a_{j} v}, j=1,2$.

\section{Langmuir equations and another form of Marchenko Lemma}

Let $R$ be a ring with unit, $\partial: R \rightarrow R$, its derivation, and $\alpha: R \rightarrow R$ an isomorphism, such that $\partial \alpha=\alpha \partial$. Let also $\Gamma, A \in R$ be invertible elements such that $\partial A=0, \partial \Gamma=\alpha^{2}(\Gamma)$, and $\partial \Gamma+\Gamma=\alpha(\Gamma) A$. Set $\gamma=(\partial \Gamma) \Gamma^{-1}$. Since $\alpha$ is an isomorphism, $\gamma$ is invertible. The following lemma was proved in $[\mathrm{M}]$.

Lemma 7. The element $U=\gamma \alpha^{-1}(\gamma)^{-1}=1+\alpha(\gamma)-\gamma$ satisfies the following equality:

$$
\partial U=\alpha(U) U-U \alpha^{-1}(U)
$$


Proof. We will show first that

$$
\begin{gathered}
(\alpha(\gamma)-\gamma)(\gamma+1)=\partial \gamma \\
\alpha(\alpha(\gamma)-\gamma) \gamma=\alpha(\gamma)-\gamma
\end{gathered}
$$

Note, that $\partial \gamma=\left(\partial^{2} \Gamma\right) \Gamma^{-1}-\gamma^{2}$. From here one has

$$
\begin{aligned}
\alpha(\gamma) & =\alpha(\partial \Gamma) \alpha(\Gamma)^{-1}=\left(\partial^{2} \Gamma+\partial \Gamma\right)(\partial \Gamma+\Gamma)^{-1} \\
& =\left(\left(\partial^{2} \Gamma\right) \Gamma^{-1}+\gamma\right)(\gamma+1)^{-1}=\left(\partial \gamma+\gamma^{2}+\gamma\right)(\gamma+1)^{-1}=\partial \gamma(\gamma+1)^{-1}+\gamma
\end{aligned}
$$

This leads to (10). To prove (11), we notice that

$$
\begin{aligned}
\alpha^{2}(\gamma) & =\alpha^{2}(\partial \Gamma) \alpha^{2}(\Gamma)^{-1}=\partial\left(\alpha^{2}(\Gamma)\right) \alpha^{2}(\Gamma)^{-1}=\partial^{2}(\Gamma)(\partial \Gamma)^{-1} \\
& =\left(\partial^{2} \Gamma\right) \Gamma^{-1} \gamma^{-1}=\left(\partial \gamma+\gamma^{2}\right) \gamma^{-1}=(\partial \gamma) \gamma^{-1}+\gamma
\end{aligned}
$$

Then

$$
\left(\alpha^{2}(\gamma)-\alpha(\gamma)\right) \gamma=\partial \gamma+\gamma^{2}-\alpha(\gamma) \gamma
$$

By (10) the last expression equals to $\alpha(\gamma)-\gamma$ and (11) is proved. Note that $\alpha(U) \gamma=\alpha(\gamma)$ and (10) implies

$$
U=1+\alpha(\gamma)-\gamma
$$

Together with (10) it gives us $U \alpha^{-1}(U)=1+\partial \gamma$. From (10) it follows that $\partial(\alpha \gamma)=\alpha^{3}(\gamma)-\alpha(\gamma)$. Together with (11) it implies $\alpha(U) U=1+\partial(\alpha \gamma)$. From (12) it follows that

$$
\partial U=\partial(\alpha \gamma)-\partial(\gamma)=\alpha(U) U-U \alpha^{-1}(U) .
$$

Similarly to subsection 3 we will consider the following specialization of Lemma 7 . Let $(Q, \partial)$ be a differential algebra with unit. Suppose we are given two sets of elements $f_{i j}, a_{i j}, i \in \mathbb{Z}, j=1,2, \ldots, N$ in $Q$ such that for all $i, j$ $\partial a_{i j}=0$ and

$$
\partial f_{i j}=f_{i+2 j}, \partial f_{i j}+f_{i j}=f_{i+1 j} a_{i j} .
$$

Suppose also that the Wronski matrices $W_{k}=W\left(f_{k 1}, \ldots, f_{k N}\right)$ are invertible and the quasideterminants $\left|\partial W_{k}\right|_{N N}$ are defined and invertible. Set

$$
\begin{aligned}
g_{k} & =\left|\partial W_{k}\right|_{N N}\left|W_{k}\right|_{1 N}^{-1} \cdot\left|W_{k-1}\right|_{1 N}\left|\partial W_{k-1}\right|_{N N}^{-1} \\
& =1+\left|\partial W_{k+1}\right|_{N N}\left|W_{k+1}\right|_{1 N}^{-1}-\left|\partial W_{k}\right|_{N N}\left|W_{k}\right|_{1 N}^{-1} .
\end{aligned}
$$


Theorem 8. Elements $g_{k}, k \in \mathbb{Z}$ satisfy generalized Langmuir equations

$$
\partial g_{k}=g_{k+1} g_{k}-g_{k} g_{k-1} \text {. }
$$

Proof. The proof is similar to the proof of Theorem 5. Let

$$
R=Q^{\infty}=\left\{\left(w_{k}\right), k \in \mathbb{Z}\right\}
$$

be an algebra with coordinate-wise multiplication and coordinate-wise derivation induced by $\partial$. Set $\alpha\left(\left(w_{k}\right)\right)=\left(w_{k+1}\right)$. Take $w_{k}=W_{k}$ and $A=\left(A_{k}\right), A_{k}=$ $\operatorname{diag}\left(a_{k 1}, \ldots, a_{k N}\right)$. Then apply Lemma 7 and Proposition 1.

Note that in a commutative case the last equation can be written in the form

$$
\partial\left(\log g_{k}\right)=g_{k+1}-g_{k-1} .
$$

Example. Let $Q=S[[t]]$, where $S$ is an algebra with unit and $t$ and independent variable commuting with elements of $S$. Set $\partial=\partial / \partial t$. For $i \in \mathbb{Z}, j=1, \ldots, N$ consider a set of formal series

$$
f_{i j}(t)=p_{j} \mu_{j}^{i} e^{\mu_{j}^{2} t}+q_{j} \mu_{j}^{-i} e^{\mu_{j}^{-2} t}
$$

with $p_{j}, q_{j}, \mu_{j} \in S$. These series satisfy equations (13) with $a_{i j}=\mu_{j}+\mu_{j}^{-1}$. Let $N=1$. Omitting the index $j=1$ we get that functions

$$
\begin{aligned}
g_{k}= & \left(q+p \mu^{2 k+4} e^{\left(\mu^{2}-\mu^{-2}\right) t}\right) \mu^{-2}\left(q+p \mu^{2 k} e^{\left(\mu^{2}-\mu^{-2}\right) t}\right)^{-1} \\
& \times\left(q+p \mu^{2 k-2} e^{\left(\mu^{2}-\mu^{-2}\right) t}\right) \mu^{2}\left(q+p \mu^{2 k+2} e^{\left(\mu^{2}-\mu^{-2}\right) t}\right)^{-1}
\end{aligned}
$$

satisfy the generalized Langmuir equations.

Remark. To obtain a solution of the periodic Langmuir equations one has to take $\mu_{j}$ 's to be the corresponding roots of 1 .

\section{Nonlinear Schrödinger equation}

We will use the following form of the Marchenko Lemma. Let $R$ be an algebra with unit and let $\partial, \partial_{0}, \partial_{1}$ be its derivations commuting with each other. Let elements $A, B, C$ belong to the common kernel of all three derivations and consider an invertible element $\Gamma \in R$ such that

$$
\begin{gathered}
\partial_{0} \Gamma+B \partial^{2} \Gamma=\Gamma C \\
\partial_{1} \Gamma+B \partial \Gamma=\Gamma A .
\end{gathered}
$$

The following lemma can also be found in [M]. 
NONABELIAN INTEGRABLE SYSTEMS

Lemma 9. Let

$$
\begin{aligned}
\gamma & =(\partial \Gamma) \Gamma^{-1} \\
U & =\gamma B-B \gamma \\
V & =\gamma B+B \gamma
\end{aligned}
$$

If $B^{2}=1$ then $U$ and $V$ satisfy the following equations

$$
\begin{gathered}
2 B \partial_{0} U+\left(\partial_{1}^{2}+\partial^{2}\right) U+2 U^{3}-2\left(U \partial_{1} V+\left(\partial_{1} V\right) U\right)=0, \\
\partial_{1} V+B \partial V=U^{2} .
\end{gathered}
$$

Proof. Multiply (14) by $\Gamma^{-1}$ from the left, apply the derivation $\partial$, and then multiply by $\Gamma$ from the left. Then we get

$$
-\gamma \partial_{0} \Gamma+\partial_{0} \partial \Gamma-\gamma B \partial^{2} \Gamma+B \partial^{3} \Gamma=0
$$

One can easily check that

$$
\begin{aligned}
\partial_{0} \gamma & =\left(\partial_{0} \partial \Gamma\right) \Gamma^{-1}-\gamma\left(\partial_{0} \Gamma\right) \Gamma^{-1} \\
\left(\partial^{2} \Gamma\right) \Gamma^{-1} & =\partial \gamma+\gamma^{2} \\
\left(\partial^{3} \Gamma\right) \Gamma^{-1} & =\partial^{2} \gamma+2(\partial \gamma) \gamma+\gamma \partial \gamma+\gamma^{3} .
\end{aligned}
$$

The substitution of these formulas to (18) multiplied by $\Gamma^{-1}$ from the right leads us to the equality

$$
\partial_{0} \gamma+B\left(\partial^{2} \gamma+2(\partial \gamma) \gamma\right)+[B, \gamma]\left(\partial \gamma+\gamma^{2}\right)=0
$$

In a similar way (17) leads us to

$$
\partial_{1} \gamma+B \partial \gamma+[B, \gamma] \gamma=0
$$

Let us apply to (20) the derivation $\partial_{1}$. Then

$$
\partial_{1}^{2} \gamma+B \partial \partial_{1} \gamma+\left[B, \partial_{1} \gamma\right] \gamma+[B, \gamma] \partial_{1} \gamma=0
$$

Replacing in this formula $\partial_{1} \gamma$ by $-B \partial \gamma-[B, \gamma] \gamma$ and using the identity $B^{2}=1$ one has

$$
\left(\partial_{1}^{2}-\partial^{2}\right) \gamma-2\left(B[B, \partial \gamma] \gamma+B[B, \gamma] \gamma^{2}+[B, \gamma]^{2} \gamma\right)=0
$$

It allows us to exclude from (19) the term $[B, \gamma] \gamma^{2}$ and get

$$
2 \partial_{0} \gamma+B\left(\partial^{2}+\partial_{1}^{2}\right) \gamma+4 B(\partial \gamma) \gamma+2[B, \gamma] \partial \gamma-2[B, \partial \gamma] \gamma-2 B[B, \gamma]^{2} \gamma=0
$$

or

$$
2 \partial_{0} \gamma+B\left(\partial^{2}+\partial_{1}^{2}\right) \gamma+2\{B, \partial \gamma\} \gamma+2[B, \gamma](\partial \gamma-B[B, \gamma] \gamma)=0
$$


where $\{x, y\}=x y+y x$.

Multiplication of (21) by $B$ from the left and using (20) leads us to

$$
2 B \partial_{0} \gamma+\left(\partial^{2}+\partial_{1}^{2}\right) \gamma+2[B, \gamma]^{2} \gamma-2\left\{B, \partial_{1} \gamma\right\} \gamma+2[B, \gamma] \partial_{1} \gamma=0 .
$$

Commutation the last equality with $B$ gives us (16). Note also, that (20) implies (17).

We will apply Lemma 9 when $\partial_{1}=0$. It will give us a nonabelian version of the nonlinear Schrödinger equation. When $\partial_{1} \neq 0$ similar arguments lead to the nonabelian Davey-Stewartson system (cf.[LY]).

Let $Q$ be an algebra with unit and with two commuting derivations $\partial_{0}$ and $\partial$. Let $R$ be the algebra of $N \times N$ - matrices over $Q$. We extend in an obvious way the derivations $\partial_{0}$ and $\partial$ to the derivations of $R$ and denote them by the same letters. For an element $f \in Q$ denote $\partial^{k} f$ by $f^{(k)}$, and for a set $f_{1}, \ldots, f_{N}$ let

$$
W=W\left(f_{1}, \ldots, f_{N}\right)=\left(f_{j}^{(k-1)}\right), k, j=1, \ldots, N .
$$

Suppose that $W$ is invertible and that

$$
N \times N \text {-matrices } \Gamma=W, B=\operatorname{diag}\{b, \ldots, b\}, A
$$

satisfy equations (14) in $R$. Suppose also that $b^{2}=1$ and set $g=|\partial W|_{N N}|W|_{1 N}^{-1}$. Then Lemma 9 implies

Theorem 10. The element $U=g b-b g$ satisfies the equation

$$
2 b \partial_{0} U+\partial^{2} U+2 U^{3}=0 .
$$

If $Q$ itself is an algebra of $r \times r$-matrices over an algebra with unit and $b=\operatorname{diag}\{1, \ldots, 1,-1, \ldots,-1\}$ (there are $r_{1} 1$ 's and $r_{2}=r-r_{1}(-1)$ 's) then $U=\left(U_{p q}\right) p, q=1,2$ is a block-matrix where $U_{p q}$ is an $r_{p} \times r_{q}$-matrix and $U_{11}=0, U_{22}=0$. Then (22) implies

$$
\begin{aligned}
& 2 \partial_{0} U_{12}+\partial^{2} U_{12}+2 U_{12} U_{21} U_{12}=0 \\
& -2 \partial_{0} U_{21}+\partial^{2} U_{21}+2 U_{21} U_{12} U_{21}=0 .
\end{aligned}
$$

Let $Q$ is the algebra of matrices over an algebra with an involutive antiautomorphism *. Denote by $X^{*}$ the hermitian conjugation to the matrix $X$. If $r_{1}=r_{2}$ then one could choose $A$ such that $U_{21}=U_{12}^{*}$. Assume that $* \partial_{0} *=-\partial_{0}$, $* \partial *=\partial$. Then from $(23)$ we get an equation for $X=U_{12}$ :

$$
2 \partial_{0} X+\partial^{2} X+2 X X^{*} X=0 .
$$


As an example we will consider an algebra of formal series $R[[u, v]]$ over a not necessarily commutative algebra $R$ with unit over complex numbers. Here variables $u$ and $v$ commute with each other and with elements of $R$. We set $\partial_{0}=i \partial / \partial u, \partial=\partial / \partial v$, where $i=\sqrt{-1}$. Let $b^{2}=1, b \in R$, set $q_{1}=(1+b) / 2, q_{2}=$ $(1-b) / 2$. Then $b q_{1}=q_{1}, b q_{2}=-q_{2}$. Let $a_{1}, \ldots, a_{N}, c_{1}, \ldots, c_{N}, d_{1}, \ldots, d_{N} \in R$. Set

$$
f_{j}=q_{1} c_{j} e^{a_{j} v+i a_{j}^{2} u}+q_{2} d_{j} e^{-a_{j} v-i a_{j}^{2} u}, j=1, \ldots, N .
$$

It is easy to see that $W=W\left(f_{1}, \ldots, f_{N}\right)$ satisfies equations (14) with $C=$ $0, A=\operatorname{diag}\left\{a_{1}, \ldots, a_{N}\right\}$.

As an example, we consider case $N=1$. Then $U=g b-b g$ where

$$
g=\left(q_{1} c e^{a v+i a^{2} u}+q_{2} d e^{-a v-i a^{2} u}\right) a\left(q_{1} c e^{a u+i a^{2} v}+q_{2} d e^{-a v-i a^{2} u}\right)^{-1} .
$$

is a solution of equation (22).

One may consider a case when $R$ is the algebra of $2 \times 2$-matrices over a $\mathbb{C}$ algebra $R_{0}$ with an involutive antiautomorphism $a \rightarrow a^{*}$ which agrees with the complex conjugation. For appropriate $c$ and $d f \in R[[u, v]]$ could be written as

$$
f=\left(\begin{array}{cc}
\alpha e^{x} & \beta^{*} e^{-x^{*}} \\
\beta e^{-x} & \alpha^{*} e^{x^{*}}
\end{array}\right)
$$

where $x=a v+i a^{2} u$, and $a, \alpha, \beta \in R_{0}$. Then $\Gamma=f$ satisfies equations (14) with $C=0, B=\operatorname{diag}\{1,-1\}, A=\operatorname{diag}\left\{a,-a^{*}\right\}$. Consider the $2 \times 2$-matrix $\left(\gamma_{p q}\right)=(\partial f / \partial v) f^{-1}$. One can see that $\gamma_{21}=\gamma_{12}^{*}$. It follows that

$$
w=-2 \gamma_{12}=2\left(\alpha a \alpha^{-1}+\beta^{*} a^{*}\left(\beta^{*}\right)^{-1}\right)\left(\alpha^{*} e^{2 x^{*}}\left(\beta^{*}\right)^{-1}-\beta e^{2 x} \alpha^{-1}\right)^{-1}
$$

satisfies the equation

$$
2 \partial_{0} w+\partial^{2} w+2 w w^{*} w=0 .
$$

When $R_{0}=\mathbb{C}$ one could write $\alpha e^{2 x} \beta^{-1}=e^{y}$ and then arrive to a well-known example $[\mathrm{LS}]$

$$
w=(a+\bar{a}) e^{-i I} / \sinh R,
$$

where $I=\operatorname{Im} y, R=\operatorname{Re} y$.

Remark. One could change the variable $U \rightarrow i U$ in the Schrödinger equation and arrive to the nonlinear heat equation. Then we could eliminate $i=\sqrt{-1}$ in our formulas and to generalize them to the case of any field of characteristic zero. 


\section{References}

[EGR] P. Etingof, I. Gelfand, and V. Retakh, Factorization of differential operators, quasideterminants, and nonabelian Toda field equations, Math. Res. Lett. 4 (1997), 413-425.

[GR1] I. Gelfand and V. Retakh, Determinants of matrices over noncommutative rings, Funct. Anal. Appl. 25 (1991), no. 2, 91-102.

[GR2] - A theory of noncommutative determinants and characteristic functions of graphs, Funct. Anal. Appl. 26 (1992), no. 4, 1-20.

[GR3] A theory of noncommutative determinants and characteristic functions of graphs.I, in: Publ. LACIM, UQAM 14 (1993), 1-26.

[GR4] , Quasideterminants, I, Selecta Math. (to appear).

[Kr] I. M. Krichever, The periodic non-abelian Toda chain and its two-dimensional generalization, Russ. Math. Surv. 36 (1981), no. 2, 82-89.

[KN] I. M. Krichever and S. P. Novikov, Holomorphic bundles over algebraic curves and nonlinear equations, Russ. Math. Surv. 35 (1980), no. 6, 53-79.

[LS] A. N. Leznov and M. V. Saveliev, Group methods of integration of nonlinear dynamical systems, (in Russian), Nauka, Moscow, 1985.

[LY] A. N. Leznov and E. A. Yuzbashyan, Multi-soliton solutions of Two-dimensional Davey-Stewartson equation, hep-th 9612107.

[M] V. A. Marchenko, Nonlinear equations and operator algebras, (in Russian), Naukova Dumka, Kiev, 1986.

[P] J. H. H. Perk, Equations of motions for the transverse correlations of the one-dimensional XYZ-model at finite temperature, Phys. Lett. A 92 (1980), 1-5.

[PC] J. H. H. Perk and H. W. Capel, Transverse correlations in the inhomogeneous XYmodel at infinite temperature, Physica A 92 (1978), 163-184.

[RS] A. V. Razumov and M. V. Saveliev, Maximally nonabelian Toda systems, Nuclear Physics B, hep-th 9612081.

Department of Mathematics, Harvard University, Cambridge, MA 02138

E-mail address: etingof@math.harvard.edu

Department of Mathematics, Rutgers University, New Brunswick, NJ 08903

E-mail address: igelfand@math.rutgers.edu

Department of mathematics, University of Arkansas, Fayetteville, AR 72701

E-mail address: vretakh@comp.uark.edu 